Supporting information to

\title{
Elucidation of anionic and cationic redox reactions in a prototype sodium layered oxide cathode
}

Chen Cheng, ${ }^{1+}$ Siyuan Li, ${ }^{2+}$ Tiefeng Liu, ${ }^{2}$ Yujian Xia,,${ }^{1}$ Lo-Yueh Chang, ${ }^{1}$ Yingying Yan, ${ }^{1}$ Manling Ding, ${ }^{1}$ Yue $\mathrm{Hu},{ }^{1}$ Jinpeng $\mathrm{Wu},{ }^{3 *}$ Jinghua Guo, ${ }^{3,4^{*}}$ and Liang Zhang ${ }^{1 *}$

1. Institute of Functional Nano \& Soft Materials (FUNSOM), Jiangsu Key Laboratory for Carbon-Based Functional Materials \& Devices, Joint International Research Laboratory of Carbon-Based Functional Materials and Devices, Soochow University, 199 Ren'ai Road, Suzhou 215123, Jiangsu, China

2. College of Materials Science and Engineering, Zhejiang University of Technology, Hangzhou 310014, Zhejiang, China.

3. Advanced Light Source, Advanced Light Source, Lawrence Berkeley National Laboratory, Berkeley, California 94720, USA

4. Department of Chemistry and Biochemistry, University of California, Santa Cruz, California 95064, USA

${ }^{+}$These two authors contributed equally to this work.

*Email: liangzhang2019@suda.edu.cn (Liang Zhang); jguo@lbl.gov (Jinghua Guo); jinpeng@lbl.gov (Jinpeng Wu) 


\section{Experimental details}

\section{Cell preparation and electrochemical cycling}

P2-type $\mathrm{Na}_{2 / 3} \mathrm{Ni}_{1 / 3} \mathrm{Mn}_{2 / 3} \mathrm{O}_{2}$ (NaNMO) powders were synthesized through a typical solid-state reaction process. Raw materials of $\mathrm{Na}_{2} \mathrm{CO}_{3}$ (Aladdin), $\mathrm{NiO}$ (Aladdin), and $\mathrm{MnO} \cdot 4 \mathrm{H}_{2} \mathrm{O}$ (Aladdin) are weighed out according to the stoichiometric amounts of NaNMO. The resultant mixtures were ground using a mortar and pestle and then ball-milled in a planetary zirconia ball miller at room temperature at a speed of $400 \mathrm{rpm}$ for $6 \mathrm{~h}$. The obtained powders were dried at $80^{\circ} \mathrm{C}$ overnight and then calcined at $920^{\circ} \mathrm{C}$ in compressed air for $12 \mathrm{~h}$. As-prepared NaNMO powder were stored in a glove box filled with Ar to avoid contamination by moisture.

\section{Electrochemical measurements}

The NaNMO powder, super $\mathrm{P}$, and PVDF binder at a weight ratio of $8: 1: 1$ were mixed in a $\mathrm{N}$ methylpyrrolidone (NMP) solvent to form a homogeneous slurry. The obtained slurry is coated on slurry was plastered on an $\mathrm{Al}$ foil and then dried at $100{ }^{\circ} \mathrm{C}$ overnight in a vacuum oven to remove the NMP. The mass loading of the electrodes is $1.0-1.5 \mathrm{mg} / \mathrm{cm}^{2}$ with a thickness of $15-20 \mu \mathrm{m}$ and the porosity of the electrodes is $30-40 \%$. The working electrode was fabricated by cutting out round disks with a $14 \mathrm{~mm}$ diameter. CR2325 coin cells were assembled in an argon-filled glove box using metallic sodium foil as the counter/reference electrode, glass fiber paper as the separator, and $1 \mathrm{M}$ $\mathrm{NaClO}_{4}$ dissolved in a mixture of ethylene carbonate and propylene carbonate with a volume ratio of $1: 1$ as the electrolyte. The galvanostatic charge/discharge testing was performed on a LAND

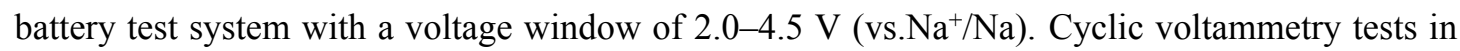
the voltage window of $2.0-4.5 \mathrm{~V}$ were carried out at a scan rate of $0.1 \mathrm{mV} \mathrm{s}^{-1}$. All the cells were tested at room temperature. 


\section{Morphology and crystal structure characterizations}

Powder X-ray diffraction (PXRD) measurements were conducted on a Rigaku AXS D8 diffractometer with $\mathrm{Cu} \mathrm{Ka}$ radiation. The morphology of the samples was examined by a field emission scanning electron microscope (SEM, Utral 55) equipped with an energy dispersive spectroscopy (EDS) detector.

\section{Soft X-ray experiments}

The sXAS and mRIXS measurements were performed at beamline 8.0.1 of the Advanced Light Source (ALS), beamline 02B02 of the Shanghai Synchrotron Radiation Facility, and beamline 20A of Taiwan Light Source. ${ }^{1}$ The electrodes at different states of charge were disassembled and rinsed with dimethyl carbonate thoroughly to remove the surface residue. Specifically, we used a homemade suitcase to directly transfer the samples from glovebox to beamline without the exposure to air to avoid the change of oxidation states of cycled electrodes. ${ }^{3}$ The XAS spectra in TFY and TEY mode were first normalized by the incoming beam intensity and then normalized to the absorption pre- and post-edges. The mRIXS data were collected through the ultra-high-efficiency modular spectrometer at beamline 8.0.1 with an excitation energy step of $0.2 \mathrm{eV}$. The final $2 \mathrm{D}$ images were achieved through a data process involving normalization to the beam flux and collection time, integration, and combination. Mn L-edge iPFY are obtained using the method described in our previous report. ${ }^{2}$ The quantitative fittings of Mn L-edge iPFY and Ni L-edge TFY were performed using a linear combination method with the standard experimental spectra of $\mathrm{Mn}^{2+/ 3+/ 4+}$ and $\mathrm{Ni}^{2+/ 3+/ 4+}$, respectively. ${ }^{3}$ It should be mentioned that the thickness of electrode materials is much larger than the detection depth of TEY (several nanometers) and TFY/iPFY (about $150 \mathrm{~nm}$ ). Therefore, the spectra difference should mainly come from the different reaction products of electrode surface (e.g., 
solid-electrolyte interphase species) and bulk rather than transport through the thickness of the electrode. In addition, to exclude to influence of X-ray exposure effect on our samples, we did some control experiments by changing X-ray beam flux (Figure S3). The results clearly show that no features related to oxidized oxygen states are observed even with high X-ray flux, indicating that X-ray exposure does not influence anion redox for NaNMO system.

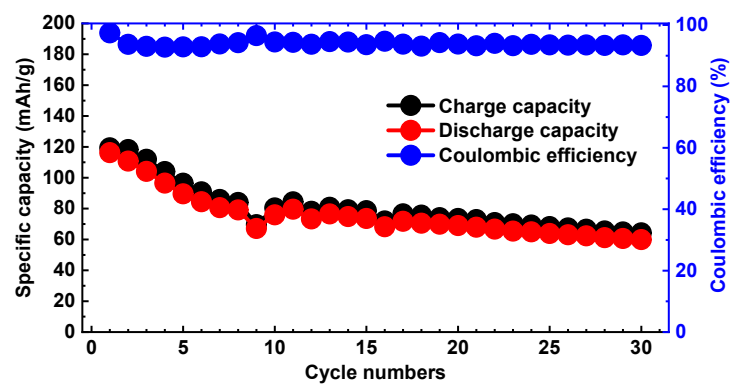

Figure S1. Capacity retention and Coulomb efficiency of NaNMO with $0.5 \mathrm{C}$ cycling rate.
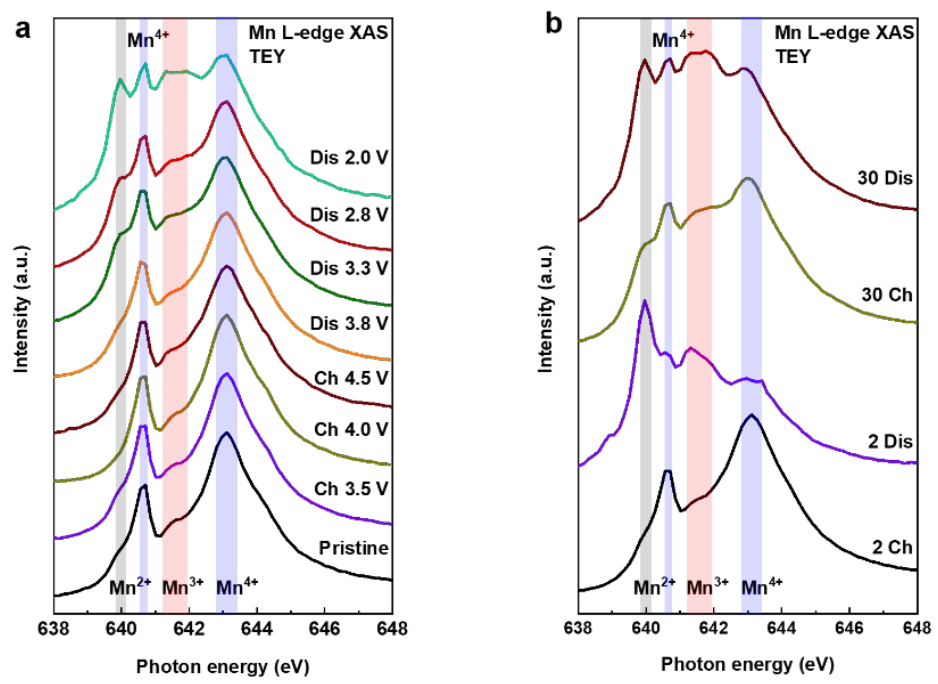

Figure S2. Mn L-edge TFY XAS spectra NaNMO electrodes at (a) different electrochemical potentials of the first cycle, and (b) fully charged and discharged states after the 2nd and 30th cycles. 

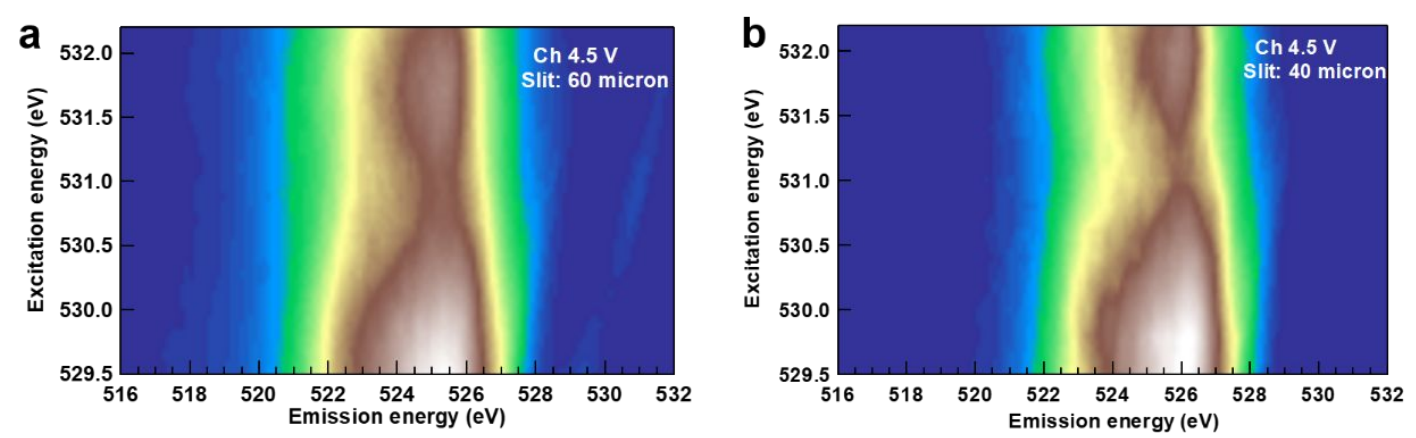

Figure S3. The O K-edge mRIXS images of NaNMO charged to $4.5 \mathrm{~V}$ measured using high (a) and low (b) X-ray flux. No features related to oxidized oxygen states are observed even with high Xray flux, indicating that X-ray exposure does not influence anion redox for NaNMO system
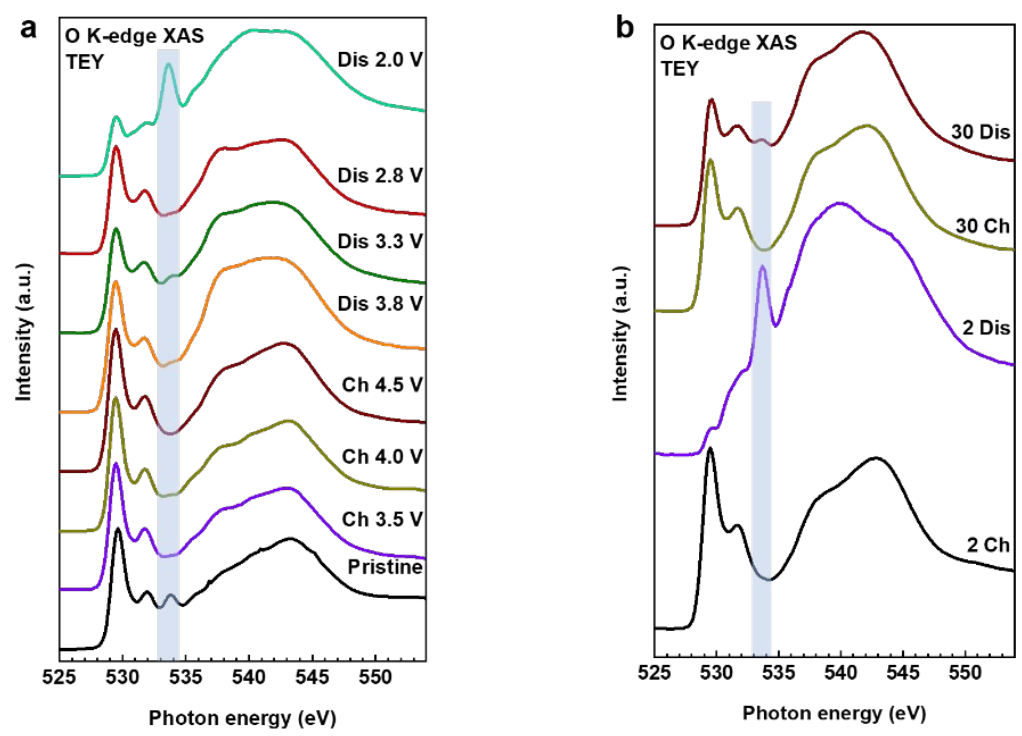

Figure S4. O K-edge TEY XAS spectra of NaNMO electrodes at (a) different electrochemical potentials of the first cycle, and (b) fully charged and discharged states after the 2nd and 30th cycles. The fingerprint feature of $\mathrm{CO}_{3}^{2-}$ is highlighted in each figure. 

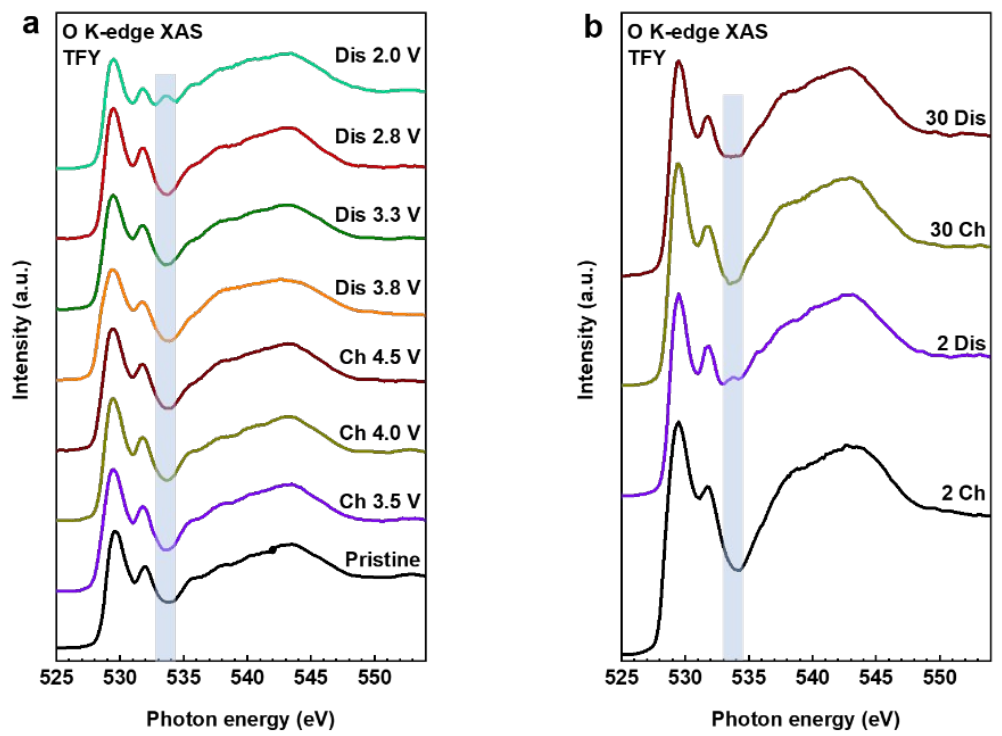

Figure S5. O K-edge TFY XAS spectra of NaNMO electrodes at (a) different electrochemical potentials of the first cycle, and (b) fully charged and discharged states after the 2nd and 30th cycles. The fingerprint feature of $\mathrm{CO}_{3}^{2-}$ is highlighted in each figure.

\begin{tabular}{|c|c|c|c|c|c|c|}
\hline & $\mathrm{Mn}^{2+}$ & Std & $\mathbf{M n}^{3+}$ & Std & $\mathbf{M n}^{4+}$ & Std \\
\hline Pristine & 0 & 0.4 & 0 & 0.7 & 100 & 0.6 \\
\hline Ch 3.5 V & 0 & 0.8 & 0 & 1.7 & 100 & 1.4 \\
\hline $\mathrm{Ch} 4.0 \mathrm{~V}$ & 0 & 5.0 & 0 & 1.0 & 100 & 8.7 \\
\hline Ch $4.5 \mathrm{~V}$ & 0 & 3.0 & 2 & 6.0 & 98 & 5.1 \\
\hline Dis $3.8 \mathrm{~V}$ & 0 & 3.8 & 0 & 7.5 & 100 & 6.5 \\
\hline Dis $3.3 \mathrm{~V}$ & 0 & 2.7 & 0 & 5.5 & 100 & 4.7 \\
\hline Dis $2.8 \mathrm{~V}$ & 0 & 2.5 & 2 & 5.1 & 98 & 4.4 \\
\hline Dis $2.0 \mathrm{~V}$ & 3 & 3.1 & 15 & 6.4 & 82 & 5.5 \\
\hline
\end{tabular}

Table S1. Mn valence percentage and the corresponding standard deviation by fitting from iPFY spectra upon the 1st cycle. Std is an abbreviation for standard deviation. 


\begin{tabular}{|c|c|c|c|c|c|c|}
\hline & $\mathrm{Ni}^{2+}$ & Std & $\mathrm{Ni}^{3+}$ & Std & $\mathrm{Ni}^{4+}$ & Std \\
\hline Pristine & 98 & 0.03 & 2 & 0.04 & 0 & 0.04 \\
\hline Ch 3.5 V & 85 & 0.03 & 15 & 0.03 & 0 & 0.03 \\
\hline $\mathrm{Ch} 4.0 \mathrm{~V}$ & 75 & 0.05 & 25 & 0.05 & 0 & 0.05 \\
\hline Ch 4.5 V & 63 & 0.04 & 37 & 0.05 & 0 & 0.05 \\
\hline Dis $3.8 \mathrm{~V}$ & 95 & 0.02 & 5 & 0.02 & 0 & 0.03 \\
\hline Dis $3.3 \mathrm{~V}$ & 96 & 0.02 & 2 & 0.02 & 2 & 0.02 \\
\hline Dis $2.8 \mathrm{~V}$ & 100 & 0.04 & 0 & 0.05 & 0 & 0.05 \\
\hline Dis $2.0 \mathrm{~V}$ & 100 & 0.03 & 0 & 0.03 & 0 & 0.03 \\
\hline
\end{tabular}

Table S2. Ni valence percentage and the corresponding standard deviation by fitting from TFY spectra upon the 1 st cycle.

\begin{tabular}{|l|c|c|c|c|c|c|}
\hline & $\mathrm{Mn}^{2+}$ & Std & $\mathrm{Mn}^{3+}$ & Std & $\mathrm{Mn}^{4+}$ & Std \\
\hline 2 Ch & 0 & 3.6 & 1 & 7.3 & 99 & 6.3 \\
\hline 2 Dis & 5 & 2.6 & 15 & 5.3 & 80 & 4.5 \\
\hline 30 Ch & 4 & 2.9 & 16 & 5.9 & 80 & 5.1 \\
\hline 30 Dis & 6 & 3.1 & 25 & 6.3 & 69 & 5.5 \\
\hline
\end{tabular}

Table S3. Mn valence percentage and the corresponding standard deviation by fitting from iPFY spectra upon extended cycles.

\begin{tabular}{|l|c|c|c|c|c|c|}
\hline & $\mathrm{Ni}^{2+}$ & $\mathrm{Std}$ & $\mathrm{Ni}^{3+}$ & $\mathrm{Std}$ & $\mathrm{N}^{4+}$ & Std \\
\hline $2 \mathrm{Ch}$ & 89 & 0.03 & 9 & 0.03 & 2 & 0.03 \\
\hline 2 Dis & 96 & 0.02 & 1 & 0.02 & 3 & 0.02 \\
\hline 30 Ch & 100 & 0.03 & 0 & 0.04 & 0 & 0.04 \\
\hline 30 Dis & 99 & 0.01 & 0 & 0.01 & 1 & 0.01 \\
\hline
\end{tabular}

Table S4. Ni valence percentage and the corresponding standard deviation by fitting from TFY spectra upon extended cycles.

\section{References}

(1) Wu, J.; Sallis, S.; Qiao, R.; Li, Q.; Zhuo, Z.; Dai, K.; Guo, Z.; Yang, W. Elemental-sensitive Detection of the Chemistry in Batteries through Soft X-ray Absorption Spectroscopy and Resonant 
Inelastic X-ray Scattering. Journal of Visualized Experiments 2018, 134, e57415.

(2) Dai, K.; Wu, J.; Zhuo, Z.; Li, Q.; Sallis, S.; Mao, J.; Ai, G.; Sun, C.; Li, Z.; Gent, W. E.; Chueh, W. C.; Chuang, Y.-d.; Zeng, R.; Shen, Z.-x.; Pan, F.; Yan, S.; Piper, L. F. J.; Hussain, Z.; Liu, G.; Yang, W. High Reversibility of Lattice Oxygen Redox Quantified by Direct Bulk Probes of Both Anionic and Cationic Redox Reactions. Joule 2018, 3 , 1-24.

(3) Li, Q.; Qiao, R.; Wray, L. A.; Chen, J.; Zhuo, Z.; Chen, Y.; Yan, S.; Pan, F.; Hussain, Z.; Yang, W. Quantitative Probe of the Transition Metal Redox in Battery Electrodes through Soft X-ray Absorption Spectroscopy. J. Phys. D: Appl. Phys. 2016, 49 , 413003. 